\title{
The metabolic pattern of hepatotoxic pyrrolizidine alkaloids in liver cells
}

\section{- Supporting Information -}

Ina Geburek ${ }^{1} \dagger$, Lukas Rutz ${ }^{2} \dagger$, Lan Gao ${ }^{2}$, Jan-Heiner Küpper ${ }^{3}$, Anja These ${ }^{1}$, Dieter Schrenk ${ }^{2 *}$

AUTHOR INFORMATION

\section{Corresponding Author}

* Corresponding author Email: schrenk@rhrk.uni-kl.de

Author Contributions

†These authors contributed equally.

${ }^{1}$ German Federal Institute for Risk Assessment, Department Safety in the Food Chain, MaxDohrn-Straße 8-10, 10589 Berlin, Germany

${ }^{2}$ University of Kaiserslautern, Food Chemistry and Toxicology, Erwin-Schrödinger-Straße 52, 67663 Kaiserslautern, Germany

${ }^{3}$ Molecular Cell Biology, Brandenburg University of Technology, Senftenberg, Germany

\section{Keywords}

Pyrrolizidine alkaloids, metabolic pattern, primary rat hepatocytes, HepG2 cells, genotoxicity 


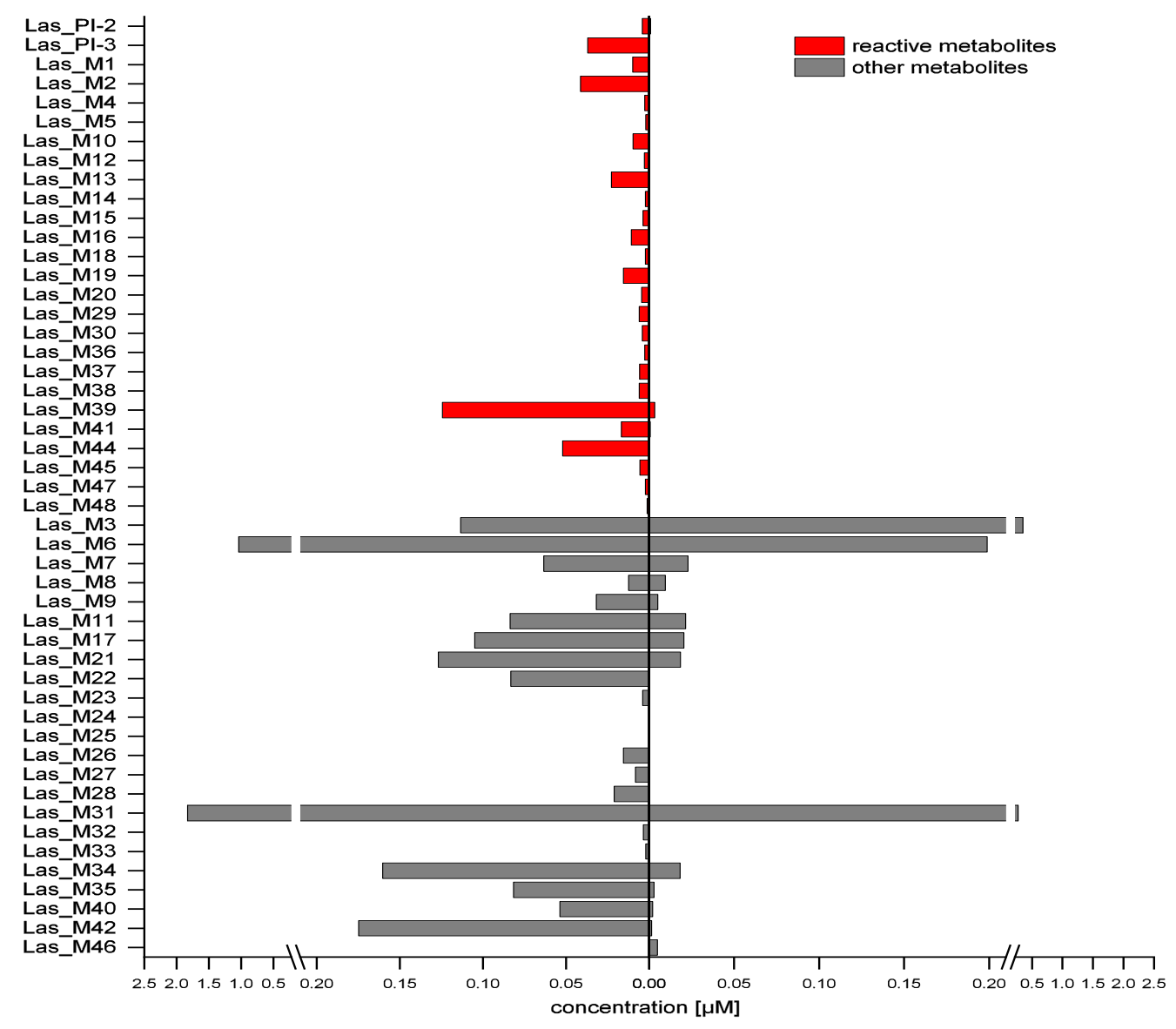

Figure S1: Amount of detected metabolites after incubation of $15 \mu \mathrm{M}$ lasiocarpine with rat liver microsomes for 60 minutes $^{1}$ (left side) and after incubation of $2.5 \mu \mathrm{M}$ lasiocarpine with primary rat hepatocytes for 24 hours (right side). Reactive metabolites are shown in red, all other metabolites are shown in gray. ${ }^{1}$ Data taken from Geburek et al. ${ }^{43}$ 


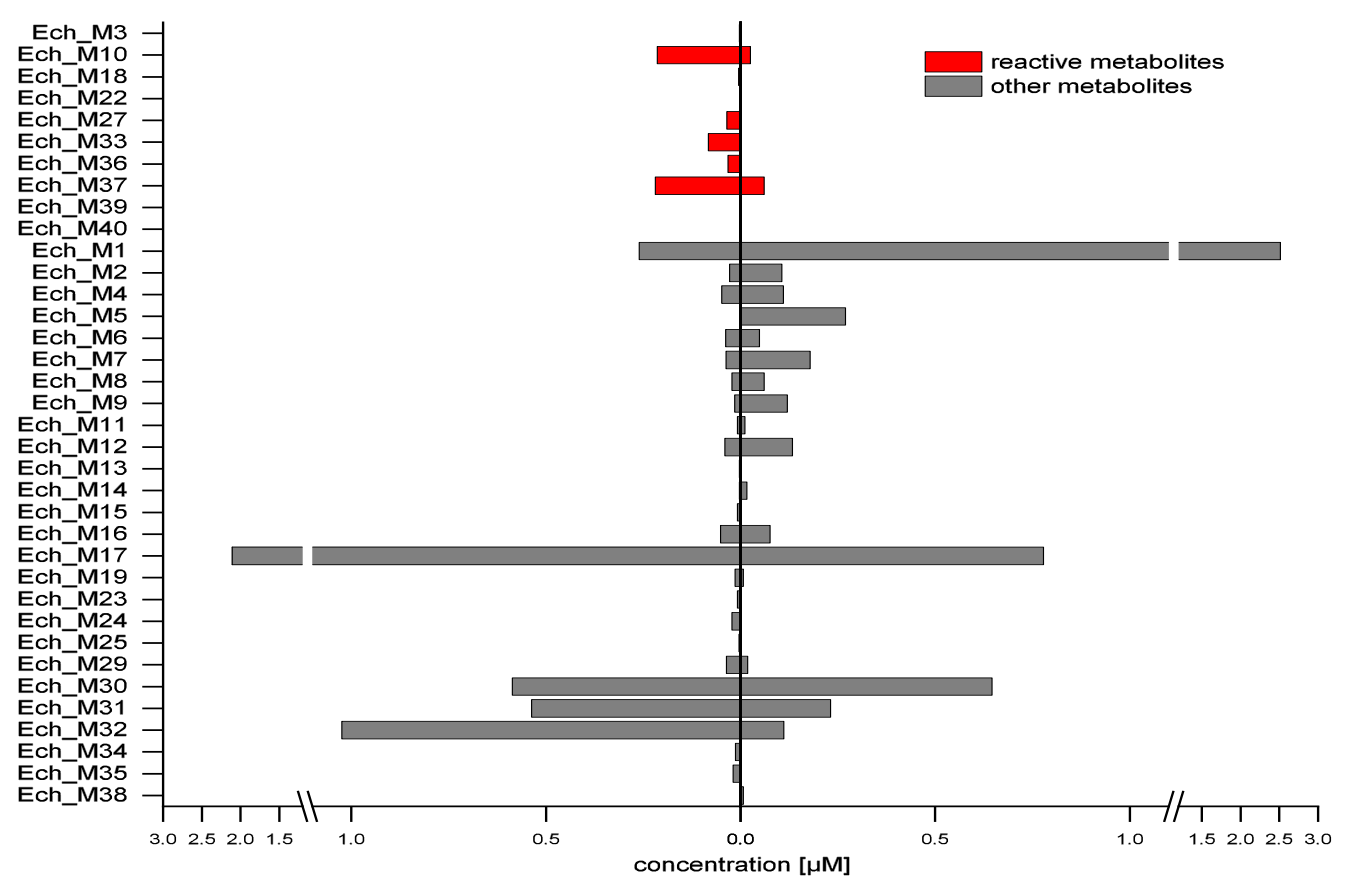

Figure S2: Amount of detected metabolites after incubation of $15 \mu \mathrm{M}$ echimidine with rat liver microsomes for 60 minutes $^{1}$ (left side) and after incubation of $25 \mu \mathrm{M}$ echimidine with primary rat hepatocytes for 24 hours (right side). Reactive metabolites are shown in red, all other metabolites are shown in gray. ${ }^{1}$ Data taken from Geburek et al. ${ }^{43}$

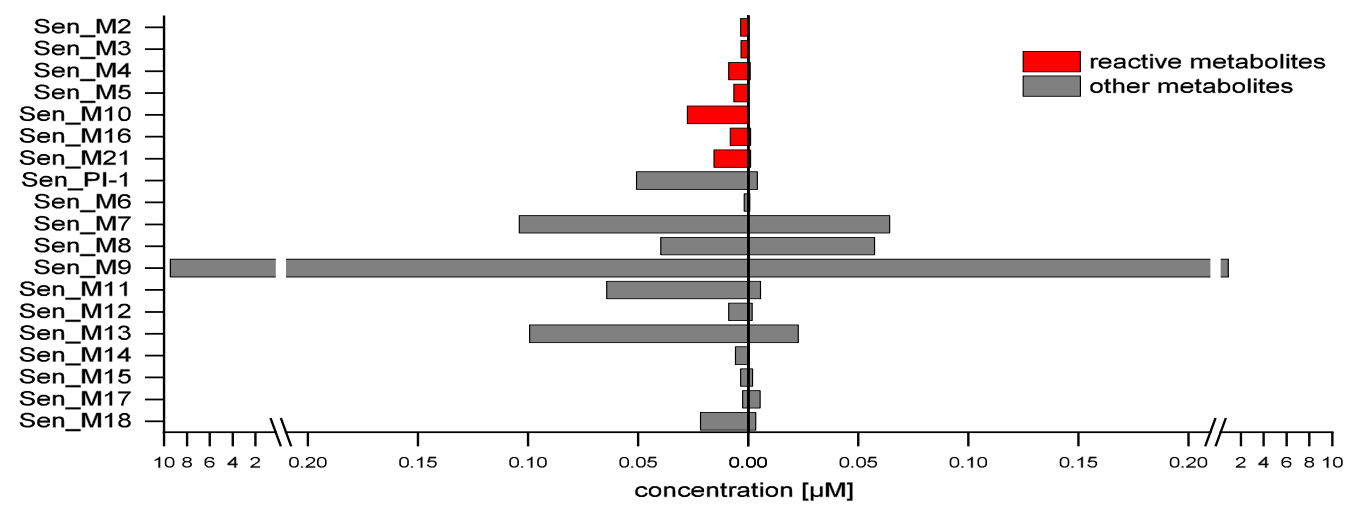

Figure S3: Amount of detected metabolites after incubation of $15 \mu \mathrm{M}$ senecionine with rat liver microsomes for 60 minutes $^{1}$ (left side) and after incubation of $2.5 \mu \mathrm{M}$ senecionine with primary rat hepatocytes for 24 hours (right side). Reactive metabolites are shown in red, all other metabolites are shown in gray. ${ }^{1}$ Data taken from Geburek et al. ${ }^{43}$

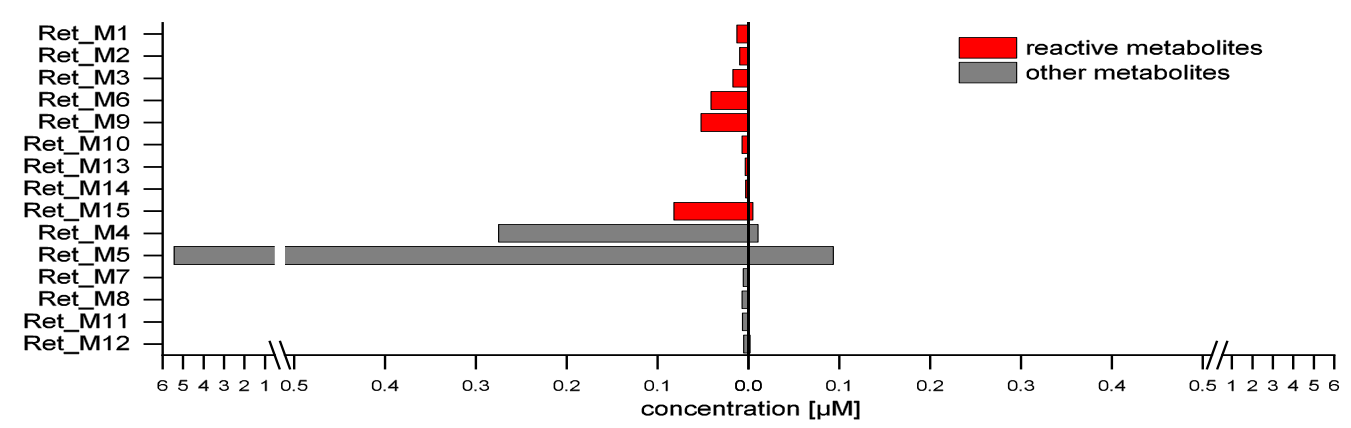

Figure S4: Amount of detected metabolites after incubation of $15 \mu \mathrm{M}$ retrorsine with rat liver microsomes for 60 minutes $^{1}$ (left side) and after incubation of $5 \mu \mathrm{M}$ retrorsine with primary rat hepatocytes for 24 hours (right side). Reactive metabolites are shown in red, all other metabolites are shown in gray. ${ }^{1}$ Data taken from Geburek et al. ${ }^{43}$ 


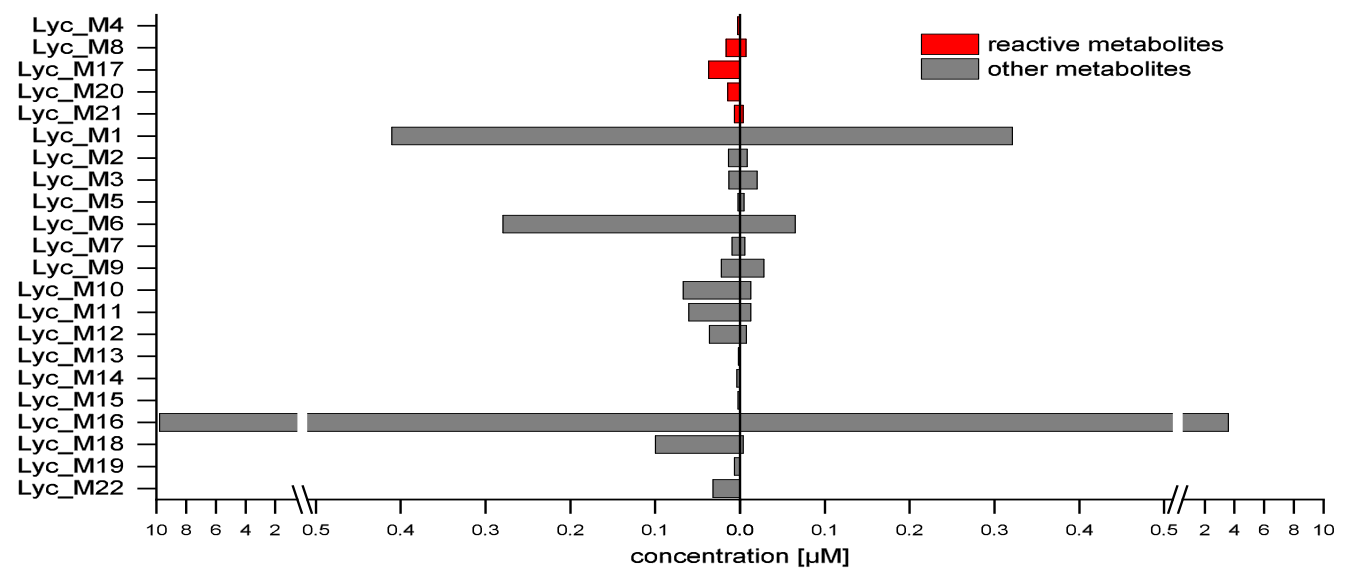

Figure S5: Amount of detected metabolites after incubation of $15 \mu \mathrm{M}$ lycopsamine with rat liver microsomes for 60 minutes $^{1}$ (left side) and after incubation of $25 \mu \mathrm{M}$ lycopsamine with primary rat hepatocytes for 24 hours (right side). Reactive metabolites are shown in red, all other metabolites are shown in gray. ${ }^{1}$ Data taken from Geburek et al. ${ }^{43}$

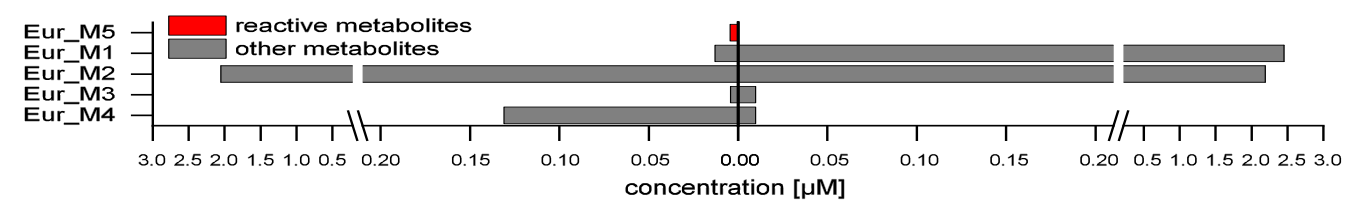

Figure S6: Amount of detected metabolites after incubation of $15 \mu \mathrm{M}$ europine with rat liver microsomes for 60 minutes ${ }^{1}$ (left side) and after incubation of $25 \mu \mathrm{M}$ europine with primary rat hepatocytes for 24 hours (right side). Reactive metabolites are shown in red, all other metabolites are shown in gray. ${ }^{1}$ Data taken from Geburek et al. ${ }^{43}$ 